\title{
The BLIiNG study - Breastfeeding length and intensity in gestational diabetes and metabolic effects in a subsequent pregnancy: a cohort study
}

\author{
Sarah J Melov ${ }^{1}$, Lisa White ${ }^{2}$, Michelle Simmons ${ }^{1}$, Adrienne Kirby ${ }^{3}$, Virginia Stulz ${ }^{4}$, Suja \\ Padmanabhan $^{5}$, Thushari Alahakoon ${ }^{1}$, Dharmintra Pasupathy ${ }^{3}$, and N Cheung ${ }^{3}$ \\ ${ }^{1}$ Westmead Hospital \\ ${ }^{2}$ Blacktown and Mount Druitt Hospital \\ ${ }^{3}$ The University of Sydney \\ ${ }^{4}$ Western Sydney University \\ ${ }^{5}$ The University of Sydney Faculty of Medicine and Health
}

September 10, 2020

\begin{abstract}
Objective To investigate if increased length and intensity of breastfeeding mediates gestational diabetes mellitus (GDM) risk in a subsequent pregnancy. Design Multisite cohort study. Setting Western Sydney, Australia, March 2017-April 2019. Population Women with a second subsequent pregnancy after a GDM-affected first pregnancy. Methods Information on breastfeeding experience, intensity and GDM management in the first pregnancy was collected by questionnaire. The results of the oral glucose tolerance test (OGTT) in the second pregnancy were also recorded. Multivariable models for OGTT and for diagnosis of GDM were fitted and then adjusted for medical treatment of GDM in the first pregnancy, BMI, age at current pregnancy and ethnicity. Main outcome measures Second pregnancy oral glucose tolerance test (OGTT) blood glucose results and diagnosis of GDM. Results We recruited 227 women with 210 eligible for analysis. Of these women, 146 (70\%) were diagnosed with recurrent GDM. We found a 19\% reduction in the risk of GDM in a subsequent pregnancy if a woman breastfed for more than six months (RR 0.81, 95\% CI 0.68-0.96) after adjusting for both age and BMI. In a fully adjusted model, the association was attenuated (RR 0.89, 95\% CI 0.78-1.02). With the same adjusted confounders, however, both high intensity breastfeeding (2 h OGTT, $\mathrm{P}=0.01)$ and breastfeeding for greater than six months $(1 \mathrm{~h}$ OGTT, $\mathrm{P}=0.01)$ were associated with a mean blood glucose decrease of $0.7 \mathrm{mmol} / \mathrm{L}$. Conclusion We found the risk of recurrent GDM was reduced by both increased duration and intensity of breastfeeding.
\end{abstract}

The BLIiNG study - Breastfeeding length and intensity in gestational diabetes and metabolic effects in a subsequent pregnancy: a cohort study

Short title: The BLIiNG Study

Sarah J Melov ${ }^{1,2}$, Lisa White ${ }^{3}$, Michelle Simmons ${ }^{4}$, Adrienne Kirby ${ }^{5}$,

Virginia Stulz $^{6}$, Suja Padmanabhan ${ }^{7,8}$, Thushari I Alahakoon ${ }^{1,2}$, Dharmintra Pasupathy ${ }^{2,9}$, N. Wah Cheung ${ }^{7,8}$

${ }^{1}$ Westmead Institute for Maternal and Fetal Medicine, Women's and Newborn Health, Westmead Hospital, Westmead, NSW, Australia

${ }^{2}$ Discipline of Obstetrics Gynaecology and Neonatology, Faculty of Medicine and Health, The University of Sydney, Sydney, NSW, Australia 
${ }^{3}$ Division of Women's and Children's, Blacktown and Mount Druitt Hospitals, Blacktown, NSW, Australia

${ }^{4}$ Women's and Newborn Health, Westmead Hospital, Westmead, NSW, Australia

${ }^{5}$ NHMRC Clinical Trials Centre, The University of Sydney, Sydney, NSW, Australia

${ }^{6}$ Western Sydney University, Nepean Hospital, Centre for Nursing \& Midwifery Research, Nepean Hospital, Penrith, NSW, Australia

${ }^{7}$ Faculty of Medicine and Health, The University of Sydney, Sydney, NSW, Australia

${ }^{8}$ Department of Diabetes and Endocrinology, Westmead Hospital, Westmead, NSW, Australia

${ }^{9}$ Department of Women and Children's Health, School of Life Course Sciences, King's College, London WC2R 2LS, England, United Kingdom

Sarah J. Melov https://orcid.org/0000-0002-7569-1016

Adrienne Kirby https://orcid.org/0000-0003-2662-4471

Virginia Stulz https://orcid.org/0000-0002-0275-8531

Suja Padmanabhan https://orcid.org/0000-0001-5418-3699

Thushari I Alahakoon https://orcid.org/0000-0002-2348-1180

Dharmintra Pasupathy https://orcid.org/0000-0002-6722-1006

N. Wah Cheung https://orcid.org/0000-0001-6323-8006

Corresponding author: Sarah J. Melov, Westmead Institute for Maternal and Fetal Medicine, Women's and Newborn Health, Westmead Hospital, Hawkesbury Rd, Westmead, NSW, Australia Ph. 0447154579 Fax. 0288904839

sarah.melov@health.nsw.gov.au

The BLIiNG study - Breastfeeding length and intensity in gestational diabetes and metabolic effects in a subsequent pregnancy: a cohort study

\section{ABSTRACT}

\section{Objective}

To investigate if increased length and intensity of breastfeeding mediates gestational diabetes mellitus (GDM) risk in a subsequent pregnancy.

\section{Design}

Multisite cohort study.

\section{Setting}

Western Sydney, Australia, March 2017-April 2019.

\section{Population}

Women with a second subsequent pregnancy after a GDM-affected first pregnancy.

\section{Methods}

Information on breastfeeding experience and GDM management in the first pregnancy was collected by questionnaire. Results of the oral glucose tolerance test (OGTT) in the second pregnancy were also recorded. Multivariable models for OGTT and for diagnosis of GDM were fitted and then adjusted for medical treatment of GDM in the first pregnancy, BMI, age at current pregnancy and ethnicity. 


\section{Main outcome measures}

Second pregnancy oral glucose tolerance test (OGTT) blood glucose results and diagnosis of GDM.

\section{Results}

We recruited 227 women with 210 eligible for analysis. Of these women, 146(70\%) were diagnosed with recurrent GDM. We found a $19 \%$ reduction in the risk of GDM in a subsequent pregnancy if a woman breastfed for more than six months (RR 0.81, 95\% CI 0.68-0.96) after adjusting for both age and BMI. In a fully adjusted model, the association was attenuated (RR 0.89, 95\% CI 0.78-1.02). With the same adjusted confounders, however, both high intensity breastfeeding $(2 \mathrm{~h} \mathrm{OGTT,} P=0.01)$ and breastfeeding for greater than six months $(1 \mathrm{~h}$ OGTT, $P=0.01$ ) were associated with a mean blood glucose decrease of $0.7 \mathrm{mmol} / \mathrm{L}$.

\section{Conclusion}

We found the risk of recurrent GDM was reduced by both increased duration and intensity of breastfeeding.

\section{Tweetable abstract}

Gestational diabetes recurrent risk is reduced by both increased duration and intensity of breastfeeding.

Funding

The authors received no specific funding for this work.

\section{Key Words}

Breastfeeding, Cardiometabolic disease, Cardiovascular disease, Diabetes, Gestational diabetes mellitus, Lactation, Postpartum, Pregnancy, Type2 diabetes mellitus, Women's health

\section{Introduction}

Gestational diabetes mellitus (GDM) is diagnosed on the basis of hyperglycemia first detected at any time during pregnancy. ${ }^{1}$ There is a global rise in the incidence of GDM, with current rates documented to be between $5-36 \%$ of pregnant women, dependent on the population, screening and diagnostic criteria. ${ }^{2-4} \mathrm{~A}$ significant individual burden is associated with GDM for women and their families including adverse perinatal outcomes, psychological impact and long-term cardiometabolic health consequences. ${ }^{5,6}$ There is a growing awareness of the rising worldwide economic implications for health service delivery associated with GDM. ${ }^{7}$ It is important to identify modifying factors that can reduce GDM and improve pregnancy outcomes for women and infants through targeted use of health resources.

The benefits of breastfeeding to infants are well documented ${ }^{8}$ and have resulted in ongoing programs worldwide to improve breastfeeding rates. However, less attention has been given to the maternal benefits. Increasing evidence suggests that breastfeeding intensity and the total length of time a woman breastfeeds during her life can positively influence her health. This includes a reduction in type 2 diabetes and an improved cardiometabolic risk profile..$^{9-14}$

Information is lacking on how breastfeeding length and intensity may impact on glycemic control and the development of GDM in a subsequent pregnancy for women who have had hyperglycemia in a prior pregnancy. Women who have GDM in their first pregnancy may have up to a 13-fold increased risk of GDM in their second pregnancy. ${ }^{15-17}$ Every subsequent pregnancy after a GDM-affected pregnancy has a compounding risk of GDM and a progression to type 2 diabetes. ${ }^{17,}{ }^{18}$ We are unaware of any research that has investigated whether breastfeeding reduces this between-pregnancy cumulative risk for the development of GDM. The aim of our breastfeeding length intensity in gestational diabetes (BLIiNG) study is to investigate women who have had GDM in their first pregnancy and assess if length and intensity of breastfeeding mediates GDM risk in a subsequent pregnancy.

\section{Methods}

Study design, study population 
This was a multisite cohort study in Western Sydney with enrolled women from four participating hospitals.

Pregnant women aged 18 years and over who had GDM in their first pregnancy and had an expected date of delivery from 1st March 2017 to 30th April 2019 were recruited. Recruitment occurred from 20 weeks' gestation. Exclusion criteria included pre-existing diabetes, pre-term first pregnancy $(<37$ weeks $)$, multiple birth first pregnancy, a history of more than three miscarriages, fetal anomaly or stillbirth. Women who had care in their first pregnancy in a country other than Australia were also excluded from the study.

All potentially eligible women were identified through the electronic maternity database from information provided at their booking visit. Eligible women were approached in the antenatal clinic or in hospital during the first two days postpartum at the four participating hospitals.

At participating hospitals, GDM was diagnosed on the basis of the 1998 Australasian Diabetes in Pregnancy Society criteria. ${ }^{19}$ This involves a $75 \mathrm{~g}$ oral glucose tolerance test (OGTT) with a fasting blood glucose level (BGL) of [?]5.5 mmol/L and/or a $2 \mathrm{~h}$ BGL [?]8.0 mmol/L. The OGTT at $1 \mathrm{~h}$ was also collected and results made available to clinicians, however, it is not routinely used for diagnostic purposes. All pregnant women were tested for GDM at 24-28 weeks' gestation, and earlier if clinically indicated. Management of GDM with insulin or metformin was defined as medical treatment inclusive of dietary modifications. Standard treatment for GDM includes blood glucose monitoring, dietician review, insulin treatment if glucose targets are not met. All women are advised to maintain a similar healthy diet after the birth of their baby and have follow-up with their family doctor.

\section{Questionnaire}

An expert panel, including lactation consultants (International Board Certified Lactation Consultants, IBCLC), endocrinologists and a maternal fetal medicine specialist, designed a task-specific, four-page health and lactation questionnaire with breastfeeding intensity assessment (Supplemental S1). Questions primarily related to the participants' previous pregnancy and were based on maternal recall. Co-design input was through a consumer trial of questionnaires, with revision from feedback, to a final questionnaire design. After revision, $92 \%(11 / 12)$ of consumers agreed or strongly agreed that the questions were easy to understand and 'accurately records how I fed my baby'.

Length of breastfeeding was assessed by a question on baby's age when all breastfeeding stopped. The Breastfeeding Length Intensity Scoring System (BLISS) was used to assess breastfeeding intensity in the first three months postpartum. The level of intensity is a determination of the amount of maternal breastfeeding inclusive of expressing, in relation to formula feeding. The highest intensity is mostly or exclusively breastfeeding, and the lowest intensity is mostly formula feeding. The scoring of intensity and determination of four levels of intensity was validated by two consultants (IBCLC) who assessed typical optimal feeding patterns and correlated the BLISS score to length of breastfeeding with a maximum score of 25. Assessment of infant feeding by BLISS occurred via maternal recall for the time periods as follows; the first week post-partum (score/6), during the first month (score/7), one to three months (score/6) and at three months (score/6). Breastfeeding only at any time point was given full marks, formula only feeding scored nil, and scores were incrementally reduced for 1-4 formula feeds a week, 1-3 per day, with four or more formula feeds per day scoring similar or equal to full formula feeding (Supplemental S1). High intensity feeding was classified as a score between 19-25, mild-moderate intensity $7-18$, and a low-intensity score was defined as between $0-6$.

\section{Data Collection}

Participating women completed the self-administered questionnaire. Women who utilised an interpreter for their obstetric appointment were verbally given the questionnaire with the assistance of the interpreter. Demographic information collected included self-identification of ethnicity, country of birth and length of time living in Australia if they were a migrant. Second pregnancy details were collected from the participating hospitals' electronic records and included booking BMI, diagnosis of GDM and pregnancy OGTT results.

Statistical Analysis 
Demographic and clinical variables were summarised between outcome groups, with percentages for categorical variables and median and interquartile range (IQR) for continuous variables, as all those included were not normally distributed. The relationships between length of breastfeeding and the BLISS score with the OGTT results from the subsequent pregnancy were analysed at each OGTT measurement time separately using linear regression. This was done after the relationship between both length of breastfeeding and BLISS score and the OGTT measurement was found to be different for each of the 3 OGTT measurements (interaction) were found to be different using a general linear model to account for the correlation within a woman. All relationships with the diagnosis of GDM were assessed with a general linear model with a log link and the results summarised as relative risks. Both length of breastfeeding and BLISS score were dichotomised for these models due to the relationships using the continuous variable not being linear. Both the models for OGTT and for diagnosis of GDM were fitted unadjusted and then adjusted for medical treatment of GDM in the first pregnancy, BMI as [?] $30 \mathrm{~kg} / \mathrm{m}^{2}$ or $<30 \mathrm{~kg} / \mathrm{m}^{2}$, age at current pregnancy [?] 30 or $<30$ years old, and ethnicity as self-identified Caucasian, South Asian or other. Family history of diabetes was assessed but did not contribute to any model so was excluded. There was no imputation of missing data and no adjustment for multiple comparisons. All analyses were done with SAS 9.4 SAS Institute Inc., Cary, NC, USA.

\section{Results}

A total of 229 eligible women were approached to participate in the BLIiNG study, with 227 women consenting to participate. Of the consenting women, 210 met all eligibility criteria and provided answers for breastfeeding intensity, with 209 women specifying length of breastfeeding. There were 210 women who had OGTT results available for their second pregnancy, $146(70 \%)$ of these women were diagnosed with recurrent GDM. Most women were born overseas with $39(19 \%)$ born in Australia. The majority of self-identified ethnicity was stated as South Asian (52\%), with Caucasian the next most frequently identified group (16\%). Demographics were similar between women diagnosed with GDM or in the non-GDM group, except for obesity, which was greater in the women with recurrent GDM (26\% vs $13 \%, P=0.03)$ (Table 1$)$.

All women in their first pregnancy commenced breastfeeding, with $117(56 \%)$ breastfeeding longer than six months and $43(20 \%)$ breastfeeding for less than three months. For the total cohort, 49 (23\%) women had low intensity BLISS score (0-6) and $36(73 \%)$ of these women breastfed for less than three months (Table 2 ). The most common reason women gave for stopping breastfeeding was "I didn't think I had enough milk" $(34 \%)$.

The relationship between previous breastfeeding duration and intensity with recurrent gestational diabetes

There were $117(56 \%)$ women who breastfed $>6$ months and of these $73(63 \%)$ developed GDM in their second subsequent pregnancy. There was an $18 \%$ reduction in the risk of recurrent GDM for any amount of breastfeeding greater than six months after the first GDM pregnancy (74/117 (63\%) vs. 71/92 (77\%), RR $0.82,95 \%$ CI $0.69-0.98, P=0.03$ ) (Table 3 ). Length of breastfeeding greater than six months remained a significant predictor for reduced GDM diagnosis when adjusted for age (RR 0.79, CI 95\% 0.67-0.94, $P$ $=0.01$ ) or BMI (RR 0.84, CI 95\% 0.70-1.00, $P=0.05$ ). After adjusting for both age and BMI there remained a $19 \%$ reduction in the risk of GDM if a woman breastfed for more than six months (RR 0.81, $95 \%$ CI $0.68-0.96, P=0.01)$. The association was attenuated in the fully adjusted model with the following confounders, medical treatment of GDM in the first pregnancy, BMI, maternal age and ethnicity (RR 0.89, 95\% CI 0.78-1.02, $P=0.09$ ) (Table 3 ).

Women who breastfed in the high intensity range ([?]19 BLISS; 63\%) had a 17\% reduction in the risk of being diagnosed with GDM compared to lower intensity breastfeeding groups, (<19 BLISS, 71/112 (63\%) vs. $75 / 98(77 \%), P=0.04$ ) (Table 3 ). In the adjusted model that included age and BMI, the association was stronger, with a $22 \%$ reduction in GDM (RR 0.78, 95\% CI 0.63-0.96, $P=0.02$ ). However, in the fully adjusted model, the association between BLISS score groups and GDM diagnosis was attenuated (RR 0.90, 95\% CI 0.77-1.05, $P=0.17$ ) (Table 3).

The relationship between previous breastfeeding duration and intensity with the glucose tolerance test result 
The relationship between breastfeeding duration and intensity with the glucose levels from the OGTT at the subsequent pregnancy were also analysed. After adjusting for confounders (medical treatment of GDM in first pregnancy, BMI, maternal age at second pregnancy, ethnicity), breastfeeding greater than six months was associated with a lower mean OGTT at $1 \mathrm{~h}(P=0.01)$ and women in the high intensity breastfeeding group had a lower $2 \mathrm{~h}$ blood glucose result $(P=0.01)$ with a mean decrease of $0.66 \mathrm{mmol} / \mathrm{L}$ (Table 4$)$.

\section{Discussion}

\section{Main findings}

Our study found that both increased duration and intensity of breastfeeding following a GDM-affected pregnancy were associated with a reduction in the risk of developing GDM in a subsequent pregnancy. Furthermore, breastfeeding duration and intensity influenced the glucose levels of the OGTT performed during the subsequent pregnancy.

There is a growing body of evidence demonstrating the association between breastfeeding and a reduction in type 2 diabetes. ${ }^{13,}$, 20, 21 Stuebe (2015) hypothesized that early, high intensity lactation may be crucial in the 'resetting' of an endocrine balance from an insulin-resistant state in pregnancy, and, without the lactationmediated reset, there may be long-term cardiometabolic health consequences. ${ }^{22}$ Studies have demonstrated that with a greater lifetime-years of maternal breastfeeding there is a corresponding risk reduction for type 2 diabetes. ${ }^{12,23}$ Research using data from the Nurses' Health Study found that for every extra lifetime-year of breastfeeding, there was a $15 \%$ reduction in the risk of type 2 diabetes for women who had a pregnancy in the last 15 years. ${ }^{20}$

In a study that included both post-GDM $(\mathrm{n}=300)$ and non-GDM $(\mathrm{n}=220)$ women, O'Reilly et al. (2011) found that hyperglycemia was more frequent at the postpartum OGTT in women who were not breastfeeding compared to women who were, with breastfeeding reducing the incidence of postpartum hyperglycemia by $60 \%{ }^{24}$ The SWIFT study was the first prospective investigation of breastfeeding intensity and hyperglycemia. ${ }^{25}$ It found an association between higher intensity breastfeeding and improved postpartum OGTT levels. As early as 6-9 weeks postpartum, a greater intensity of breastfeeding in women with GDM resulted in a lower mean fasting plasma glucose level as measured at their postpartum OGTT and a dose response to improved homeostasis model assessment (HOMA-IR). The SWIFT study also demonstrated that there was a dose-protective response of intensity and length of breastfeeding for type 2 diabetes risk two years after the GDM pregnancy. ${ }^{25}$

A lower HbA1c is associated with greater lengths of breastfeeding postpartum. ${ }^{13}$ There is also evidence that breastfeeding improves insulin sensitivity, with women who breastfed at high intensity having a lower HOMA-IR at 6-10 weeks' postpartum, though this may only be evident in obese women. ${ }^{26}$

Although there are benefits of breastfeeding for women who have had GDM, evidence suggests that these women have greater difficulty breastfeeding than unaffected women. ${ }^{27}, 28$ Women with GDM have more issues establishing breastfeeding, experience delayed lactogenesis and breastfeed for a shorter duration than other women. ${ }^{29}$ Oza-Frank et al. (2017) found that women with GDM experienced practices in hospital that are known to negatively impact on long-term breastfeeding. ${ }^{30}$ For example, they were less likely to breastfeed in the first hour after birth and it was more likely that their baby received formula in hospital than women without GDM. The importance of breastfeeding for women with GDM therefore requires greater understanding for effective, targeted lactation support. Careful consideration needs to be given to protocols whereby babies of women with gestational diabetes are routinely admitted to special care nurseries as this may negatively impact breastfeeding success. ${ }^{30}$

Breastfeeding intensity and duration can be improved through appropriate education and consistent advice that ideally includes both antenatal and postnatal care. ${ }^{31}$ Novel engagement with the use of text-based support has been trialled, with one study suggesting the best predictor for exclusive breastfeeding was strong engagement in the first two weeks postpartum utilising two-way lactation consultant texting support. ${ }^{32}$ Stuebe et al. (2016) found GDM women were less likely to stop breastfeeding or introduce formula (adjusted 
HR $0.50,95 \%$ CI $0.34-0.72$ ) at six weeks when provided with a pre- and postnatal lifestyle intervention program that included breastfeeding text-based support. ${ }^{33}$ A recent (2017) Cochrane review found that lactation support for women improved breastfeeding intensity for up to six months. ${ }^{34}$

Provision of comprehensive lifestyle and breastfeeding support for GDM women requires investment in additional staff time and resources. However, our study and others have demonstrated that optimal breastfeeding for women with a history of a GDM pregnancy reduces the risk of the mother developing type 2 diabetes and other cardiometabolic disorders. A reduction in hyperglycemia in a subsequent pregnancy also negates some of the maternal and fetal risks associated with GDM. The savings from this are likely to offset the investment in the provision of breastfeeding support. It is a financially judicious use of health resources to support early prevention for cardiometabolic disease and a unique opportunity to engage women in health intervention programs that include breastfeeding support during pregnancy and the postpartum period.

We are not aware of any published methods for the routine assessment of breastfeeding intensity. This is important as duration of breastfeeding by itself does not necessarily reflect early breastfeeding issues. Low intensity breastfeeding may occur early postpartum, however a small percentage of mothers will persist despite extreme difficulties (Table 2). Women who breastfeed more successfully with greater intensity tend to breastfeed for longer. ${ }^{35}$ The BLISS check is therefore a novel means of assessing breastfeeding intensity through a maternal recall questionnaire. Evaluation of previous breastfeeding is an important component of the pregnancy and postpartum evaluation, and this can easily be established as a routine tool for use by clinicians. The findings of our study suggest that the BLISS check score may be a useful clinical tool.

We are continuing to validate the BLISS check in a larger cohort for antenatal lactation counselling referral purposes and research. It is important to work towards standardizing the assessment of breastfeeding intensity in a manner that is clinically useful and capable of informing both care and research. Defining and standardizing breastfeeding terminology for both clinical and research purposes will assist with comparison of data and patient management. ${ }^{36}$

Strengths and limitations

The high participation rate of women gave our study strength and reduced potential recruitment bias. This suggests that women are interested in breastfeeding and are keen to discuss their breastfeeding history and associated problems. This also suggests the need for more antenatal breastfeeding support that provides an appropriate clinical avenue for breastfeeding counselling and information. Ethnic diversity is an additional strength of our study and informs the capacity to extrapolate the results to other populations.

The major weakness of our study is potential recall bias. In the literature, however, maternal recall of breastfeeding history has been validated as a reliable method to determine infant feeding history. ${ }^{37,} 38$ In a Norwegian study, recall of duration of breastfeeding was found to be accurate after 20 years with a median over-estimation of only two weeks. ${ }^{38}$ The recall bias in our BLIiNG study would be less, as all participants had only one previous pregnancy and feeding details to remember. The same recall limitation would exist for both GDM and non-GDM groups and the mean time between births was three years for both groups.

\section{Interpretation}

There is concern about the high incidence of GDM and its recurrence. Much of the focus amongst women who have had GDM is to reduce obesity and improve lifestyle in between pregnancies. However programs aimed at these risk factors are challenging, resource intensive, and have in general met with little success. ${ }^{39-41}$ On the other hand, breastfeeding is already encouraged, and for the majority of women, is acceptable, and can be undertaken successfully. Yet this has largely been neglected in the GDM and diabetes prevention literature.

Conclusions

Our study has demonstrated that a greater intensity of breastfeeding can improve glycemia and reduce recurrent GDM in pregnant women who have had a previous GDM-affected pregnancy. Breastfeeding may 
potentially reduce future type 2 diabetes and cardiovascular disease risk for the mother, as well as providing known benefits to the health of the infant. Thus, the promotion and support of breastfeeding should be routinely implemented as an important intervention for the prevention of recurrent GDM and type 2 diabetes.

Disclosure of interest

None declared. All authors declare they have no conflict of interest which may arise from being named as an author on the manuscript.

Author Contributions

The research idea was conceived by SJM and LW. The study was designed by SJM, LW, MS, IA, SP and WC. AK performed the statistical analysis, SJM interpreted the data and wrote the initial drafts with supervision from WC and DP. Invaluable support was given by IA, WC, DP, LW, MS, SP, VS and AK for final interpretation of findings, and critical revision of the article. All authors reviewed and approved the final version of the article submitted for publication.

\section{Details of ethics approval}

The study was approved by the Western Sydney Local Health District (WSLHD) Human Research and Ethics Committee (LNR/16/WMEAD/404; 1 February 2017), all participants provided informed consent.

Funding

This research did not receive any specific grant from any funding agency in the public, commercial or notfor-profit sector.

\section{Acknowledgments}

We acknowledge and appreciate the contribution to the study of data custodian Monica Hook and the assistance with data collection by Sarah Tapp.

\section{Data sharing}

No additional data is available in compliance with WSLHD Human Research and Ethics Committee regulations.

\section{References}

1. WHO recommendation on the diagnosis of gestational diabetes in pregnancy. 2016 [cited 3 March 2020]; Available from: https://extranet.who.int/rhl/topics/preconception-pregnancy-childbirth-and-postpartumcare/antenatal-care/who-recommendation-diagnosis-gestational-diabetes-pregnancy-0

2. Eades CE, Cameron DM, Evans JMM. Prevalence of gestational diabetes mellitus in Europe: A metaanalysis. Diabetes Res Clin Pract. 2017 Jul;129 : 173-81.

3. Duran A, Saenz S, Torrejon MJ, Bordiu E, Del Valle L, Galindo M, et al. Introduction of IADPSG criteria for the screening and diagnosis of gestational diabetes mellitus results in improved pregnancy outcomes at a lower cost in a large cohort of pregnant women: the St. Carlos Gestational Diabetes Study. Diabetes Care. 2014 Sep; 37 : 2442-50.

4. Lee KW, Ching SM, Ramachandran V, Yee A, Hoo FK, Chia YC, et al. Prevalence and risk factors of gestational diabetes mellitus in Asia: a systematic review and meta-analysis. BMC Pregnancy Childbirth. 2018 Dec 14; 18 : 494.

5. Kramer CK, Campbell S, Retnakaran R. Gestational diabetes and the risk of cardiovascular disease in women: a systematic review and meta-analysis. Diabetologia. 2019 Jun; 62 : 905-14. 
6. Craig L, Sims R, Glasziou P, Thomas R. Women's experiences of a diagnosis of gestational diabetes mellitus: a systematic review. BMC Pregnancy Childbirth. 2020 2020/02/07; 20 : 76.

7. Dall TM, Yang W, Halder P, Pang B, Massoudi M, Wintfeld N, et al. The Economic Burden of Elevated Blood Glucose Levels in 2012: Diagnosed and Undiagnosed Diabetes, Gestational Diabetes Mellitus, and Prediabetes. Diabetes Care. 2014; 37 : 3172-9.

8. Victora CG, Bahl R, Barros AJ, Franca GV, Horton S, Krasevec J, et al. Breastfeeding in the 21st century: epidemiology, mechanisms, and lifelong effect. Lancet. 2016 Jan 30; 387 : 475-90.

9. Kjos SL, Henry O, Lee RM, Buchanan TA, Mishell DR, Jr. The effect of lactation on glucose and lipid metabolism in women with recent gestational diabetes. Obstet Gynecol. $1993 \mathrm{Sep} ; 82$ : 451-5.

10. Ram KT, Bobby P, Hailpern SM, Lo JC, Schocken M, Skurnick J, et al. Duration of lactation is associated with lower prevalence of the metabolic syndrome in midlife - SWAN, the study of women's health across the nation. Am J Obstet Gynecol. 2008; 198 : 268.e1-.e6.

11. Schwarz EB, Ray RM, Stuebe AM, Allison MA, Ness RB, Freiberg MS, et al. Duration of lactation and risk factors for maternal cardiovascular disease. Obstet Gynecol. 2009; 113 : 974-82.

12. Gunderson EP, Hurston SR, Ning X, et al. Lactation and progression to type 2 diabetes mellitus after gestational diabetes mellitus: A prospective cohort study. Ann Intern Med. 2015; 163 : 889-98.

13. Ley SH, Chavarro JE, Li M, Bao W, Hinkle SN, Wander PL, et al. Lactation Duration and Long-term Risk for Incident Type 2 Diabetes in Women With a History of Gestational Diabetes Mellitus. Diabetes Care. 2020; 43 : 793-8.

14. Shen Y, Leng J, Li W, Zhang S, Liu H, Shao P, et al. Lactation intensity and duration to postpartum diabetes and prediabetes risk in women with gestational diabetes. Diabetes Metab Res Rev. 2019 Mar;35 : $\mathrm{e} 3115$.

15. Foster-Powell KA, Cheung NW. Recurrence of gestational diabetes. Aust N Z J Obstet Gynaecol. 1998 Nov; 38 : 384-7.

16. MacNeill S, Dodds L, Hamilton DC, Armson BA, VandenHof M. Rates and Risk Factors for Recurrence of Gestational Diabetes. Diabetes Care. 2001; 24 : 659-62.

17. Getahun D, Fassett MJ, Jacobsen SJ. Gestational diabetes: risk of recurrence in subsequent pregnancies. Am J Obstet Gynecol. 2010 Nov;203 : 467 e1-6.

18. Peters RK, Kjos SL, Xiang A, Buchanan TA. Long-term diabetogenic effect of single pregnancy in women with previous gestational diabetes mellitus. Lancet. 1996 Jan 27; 347 : 227-30.

19. Hoffman L, Nolan C, Wilson JD, Oats JJ, Simmons D. Gestational diabetes mellitus-management guidelines. The Australasian Diabetes in Pregnancy Society. Med J Aust. 1998 Jul 20; 169 : 93-7.

20. Stuebe AM, Rich-Edwards JW, Willett WC, Manson JE, Michels KB. Duration of Lactation and Incidence of Type 2 Diabetes. J Am Med Assoc. 2005; 294 : 2601-10.

21. Jager S, Jacobs S, Kroger J, Fritsche A, Schienkiewitz A, Rubin D, et al. Breast-feeding and maternal risk of type 2 diabetes: a prospective study and meta-analysis. Diabetologia. 2014 Jul;57 : 1355-65.

22. Stuebe A. Associations Among Lactation, Maternal Carbohydrate Metabolism, and Cardiovascular Health. Clin Obstet Gynecol. 2015 Dec;58 : 827-39.

23. Villegas R, Gao YT, Yang G, Li HL, Elasy T, Zheng W, et al. Duration of breast-feeding and the incidence of type 2 diabetes mellitus in the Shanghai Women's Health Study. Diabetologia. 2008 Feb; 51 : $258-66$. 
24. O'Reilly MW, Avalos G, Dennedy MC, O'Sullivan EP, Dunne F. Atlantic DIP: high prevalence of abnormal glucose tolerance post partum is reduced by breast-feeding in women with prior gestational diabetes mellitus. Eur J Endocrinol. 2011 Dec; 165 : 953-9.

25. Gunderson EP, Hedderson MM, Chiang V, Crites Y, Walton D, Azevedo RA, et al. Lactation intensity and postpartum maternal glucose tolerance and insulin resistance in women with recent GDM: the SWIFT cohort. Diabetes Care. 2012 Jan; 35 : 50-6.

26. Yasuhi I, Yamashita H, Maeda K, Nomiyama M, Mizunoe T, Tada K, et al. High-intensity breastfeeding improves insulin sensitivity during early post-partum period in obese women with gestational diabetes. Diabetes Metab Res Rev. 2019 May; 35 : e3127.

27. Matias SL, Dewey KG, Quesenberry CP, Jr., Gunderson EP. Maternal prepregnancy obesity and insulin treatment during pregnancy are independently associated with delayed lactogenesis in women with recent gestational diabetes mellitus. Am J Clin Nutr. 2014 Jan; 99 : 115-21.

28. Stuebe AM. Does breastfeeding prevent the metabolic syndrome, or does the metabolic syndrome prevent breastfeeding? Semin Perinatol. 2015 Jun; 39 : 290-5.

29. Nguyen PTH, Binns CW, Nguyen CL, Ha AVV, Chu TK, Duong DV, et al. Gestational Diabetes Mellitus Reduces Breastfeeding Duration: A Prospective Cohort Study. Breastfeed Med. 2019 Jan/Feb; 14 : 39-45.

30. Oza-Frank R, Gunderson EP. In-Hospital Breastfeeding Experiences Among Women with Gestational Diabetes. Breastfeed Med. 2017 Jun;12 : 261-8.

31. Bonuck K, Stuebe A, Barnett J, Labbok MH, Fletcher J, Bernstein PS. Effect of primary care intervention on breastfeeding duration and intensity. Am J Public Health. 2014 Feb; 104 Suppl 1: S119-27.

32. Martinez-Brockman JL, Harari N, Perez-Escamilla R. Lactation Advice through Texting Can Help: An Analysis of Intensity of Engagement via Two-Way Text Messaging. Journal of health communication $2018 ; 23: 40-51$.

33. Stuebe AM, Bonuck K, Adatorwovor R, Schwartz TA, Berry DC. A Cluster Randomized Trial of Tailored Breastfeeding Support for Women with Gestational Diabetes. Breastfeed Med. 2016 Dec; 11 : 504-13.

34. McFadden A, Gavine A, Renfrew MJ, Wade A, Buchanan P, Taylor JL, et al. Support for healthy breastfeeding mothers with healthy term babies. Cochrane Database Syst Rev. 2017.

35. Piper S, Parks PL. Use of an intensity ratio to describe breastfeeding exclusivity in a national sample. J Hum Lact. 2001 Aug; 17 : 227-32.

36. Yourkavitch J, Chetwynd EM. Toward Consistency: Updating Lactation and Breastfeeding Terminology for Population Health Research. J Hum Lact. 2019 Aug; 35 : 418-23.

37. Li R, Scanlon KS, Serdula MK. The validity and reliability of maternal recall of breastfeeding practice. Nutr Rev. 2005 Apr;63 : 103-10.

38. Natland Fagerhaug T, Forsmo S, Jacobsen GW, Midthjell K, Andersen LF, Ivar Lund Nilsen T. A prospective population-based cohort study of lactation and cardiovascular disease mortality: the HUNT study. BMC Public Health. 2013 Nov 13; 13 : 1070.

39. Koivusalo SB, Rönö K, Klemetti MM, Roine RP, Lindström J, Erkkola M, et al. Gestational Diabetes Mellitus Can Be Prevented by Lifestyle Intervention: The Finnish Gestational Diabetes Prevention Study (RADIEL): A Randomized Controlled Trial. Diabetes Care. 2016 Jan;39 : 24-30.

40. Simmons D, Jelsma JG, Galjaard S, Devlieger R, van Assche A, Jans G, et al. Results From a European Multicenter Randomized Trial of Physical Activity and/or Healthy Eating to Reduce the Risk of Gestational Diabetes Mellitus: The DALI Lifestyle Pilot. Diabetes Care. 2015 Sep;38 : 1650-6. 
41. Poston L, Bell R, Croker H, Flynn AC, Godfrey KM, Goff L, et al. Effect of a behavioural intervention in obese pregnant women (the UPBEAT study): a multicentre, randomised controlled trial. Lancet Diabetes Endocrinol. 2015; $3: 767-77$.

Table 1 Characteristics of women who were diagnosed with recurrent gestational diabetes (GDM) in their second pregnancy

\begin{tabular}{|c|c|c|c|c|c|}
\hline & & $\begin{array}{l}\text { GDM } 2^{\text {nd }} \\
\text { Pregnancy } \\
\text { No }(n=64)\end{array}$ & $\begin{array}{l}\text { GDM } 2^{\text {nd }} \\
\text { Pregnancy } \\
\text { Yes }(n= \\
146)\end{array}$ & $\begin{array}{l}\text { GDM } 2^{\text {nd }} \\
\text { Pregnancy } \\
\text { Yes }(n= \\
146)\end{array}$ & $P$-value \\
\hline $\begin{array}{l}\text { Australian- } \\
\text { born }\end{array}$ & & $16(25 \%)$ & $23(16 \%)$ & $23(16 \%)$ & 0.11 \\
\hline Self-identified & Caucasian & $14(22 \%)$ & $19(13 \%)$ & $19(13 \%)$ & 0.08 \\
\hline 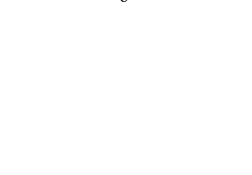 & $\begin{array}{l}\text { South Asian } \\
\text { Chinese } \\
\text { Middle East } \\
\text { Other }\end{array}$ & $\begin{array}{l}28(44 \%) \\
5(8 \%) \\
7(11 \%) \\
9(14 \%)\end{array}$ & $\begin{array}{l}81(56 \%) \\
21(14 \%) \\
6(4 \%) \\
18(12 \%)\end{array}$ & $\begin{array}{l}81(56 \%) \\
21(14 \%) \\
6(4 \%) \\
18(12 \%)\end{array}$ & \\
\hline $\begin{array}{l}\text { First degree } \\
\text { family history } \\
\text { of diabetes } \\
\text { GDM first } \\
\text { pregnancy } \\
\text { details }\end{array}$ & & $31(51 \%)$ & $80(56 \%)$ & $80(56 \%)$ & 0.47 \\
\hline $\begin{array}{l}\text { GDM- } \\
\text { medicated } 1^{\text {st }} \\
\text { pregnancy* }\end{array}$ & & $24(38 \%)$ & $69(47 \%)$ & $69(47 \%)$ & 0.19 \\
\hline $\begin{array}{l}\text { Practiced } \\
\text { cultural rest } \\
1^{\text {st }} \text { pregnancy }\end{array}$ & & $25(40 \%)$ & $72(51 \%)$ & $72(51 \%)$ & 0.14 \\
\hline $\begin{array}{l}\text { Caesarean } \\
\text { section birth } \\
1^{\text {st }} \text { pregnancy }\end{array}$ & & $39(63 \%)$ & $93(65 \%)$ & $93(65 \%)$ & 0.77 \\
\hline $\begin{array}{l}\text { Baby weight n, } \\
\text { grams, median } \\
\text { (IQR) }\end{array}$ & & $\begin{array}{l}403252 \\
(2870-3613)\end{array}$ & $\begin{array}{l}1113110 \\
(2850-3630)\end{array}$ & $\begin{array}{l}1113110 \\
(2850-3630)\end{array}$ & 0.53 \\
\hline $\begin{array}{l}\text { Baby admitted } \\
\text { Special } \\
\text { Care/NICU }\end{array}$ & & $15(24 \%)$ & $46(32 \%)$ & $46(32 \%)$ & 0.25 \\
\hline $\begin{array}{l}\text { Duration of } \\
\text { breastfeeding } \\
\text { in first } \\
\text { pregnancy } \\
\text { (months), } \\
\text { median (IQR) } \\
\text { Second } \\
\text { pregnancy } \\
\text { details }\end{array}$ & & $11(3-17)$ & $7(3-15)$ & $7(3-15)$ & 0.16 \\
\hline $\begin{array}{l}\text { Maternal age } \\
\text { (y) mean } \pm \text { SD }\end{array}$ & & $33 \pm 4$ & $33 \pm 4$ & $34 \pm 4$ & 0.04 \\
\hline
\end{tabular}


BMI at

$25(22-27)$

$25(22-27)$

$26(23-30)$

0.09

booking

median, (IQR)

BMI [?]30 at

booking

Time between

$8(13 \%)$

$8(13 \%)$

$38(26 \%)$

0.03

$3 \pm 2$

$3 \pm 2$

$3 \pm 2$

0.14

births (y)

mean \pm SD

Some variables do not equal total due to missing data

*Insulin or metformin: Insulin $(\mathrm{n}=82)$, metformin $(\mathrm{n}=10)$, both $(\mathrm{n}=1)$

Table 2

Relationship between total length of any breastfeeding and breastfeeding intensity score (BLISS)

\begin{tabular}{llll}
\hline $\begin{array}{l}\text { Length of time } \\
\text { breastfeeding }\end{array}$ & $\begin{array}{l}\text { Low BLISS } \mathbf{0}-\mathbf{6} n= \\
\mathbf{4 9} n \mathbf{( \% )}\end{array}$ & $\begin{array}{l}\text { Mild-moderate BLISS } \\
\mathbf{7 - 1 8} n=\mathbf{4 9} n \mathbf{( \% )}\end{array}$ & $\begin{array}{l}\text { High BLISS 19-25 } n \\
=\mathbf{1 1 1} n(\mathbf{\%})^{*}\end{array}$ \\
\hline$? 1$ month & $26(53)$ & $2(4)$ & $0(0)$ \\
$<3$ months & $10(20)$ & $4(8)$ & $1(1)$ \\
$3-6$ months & $9(18)$ & $22(45)$ & $18(16)$ \\
$>6-12$ months & $2(4)$ & $11(22)$ & $30(27)$ \\
$>12$ months & $2(4)$ & $10(20)$ & $62(56)$ \\
\hline
\end{tabular}

*1 length of breastfeeding missing: total 209

Table 3

Association between gestational diabetes (GDM) and length/intensity of breastfeeding

length of breastfeeding and percent of GDM

\begin{tabular}{llll}
\hline & {$[?] \mathbf{6}$ months } & {$[?] \mathbf{6}$ months } & $>\mathbf{6}$ months \\
Unadjusted & $71 / 92(77 \%)$ & $71 / 92(77 \%)$ & $74 / 117(63 \%)$ \\
Adjusted & & & \\
& Intensity of breastfeeding by BLISS score & Intensity of breastfeeding by BLISS score & Intensity of breastfees \\
& $<\mathbf{1 9}($ low- - medium $)$ & {$[?] \mathbf{1 9}($ high $)$} & {$[?] \mathbf{1 9}(\mathbf{h i g h})$} \\
Unadjusted & $75 / 98(77 \%)$ & $71 / 112(63 \%)$ & $71 / 112(63 \%)$ \\
Adjusted & & & \\
\hline
\end{tabular}

The full model adjusted for medical treatment of GDM in first pregnancy, BMI [?]30, maternal age at second pregnancy, ethnicity. BLISS: Breastfeeding Length Intensity Scoring System

Table 4

Relationship between first pregnancy length/intensity (BLISS) of breastfeeding and subsequent second pregnancy oral glucose tolerance test (OGTT results $\mathrm{mmol} / \mathrm{L}$ )

Adjusted

$\begin{array}{ll}\text { Length of breastfeeding } & \text { Length of breastfeeding } \\ \text { [?] } 6 \mathrm{mths} & >6 \mathrm{mths}\end{array}$

[?] 6mths

Mean difference $(95 \%$ CI $) \quad P$ value* 


\begin{tabular}{lllll}
\hline Adjusted & \multicolumn{3}{c}{} & \\
\hline Fasting & $4.9(0.8)$ & $4.8(0.6)$ & $0.05(-0.13,0.24)$ & 0.56 \\
$1 \mathrm{~h}$ & $10.5(2.2)$ & $9.8(1.9)$ & $0.67(0.16,1.19)$ & 0.01 \\
$2 \mathrm{~h}$ & $8.6(1.9)$ & $8.1(1.9)$ & $0.51(-0.01,1.02)$ & 0.05 \\
& Intensity of breastfeeding & Intensity of breastfeeding & & \\
& BLISS <19 & BLISS [?]19 & $0.05(-0.13,0.23)$ & 0.61 \\
Fasting & $4.9(0.8)$ & $4.8(0.6)$ & $0.40(-0.12,0.93)$ & 0.13 \\
$1 \mathrm{~h}$ & $10.4(2.3)$ & $9.8(1.8)$ & $0.66(0.15,1.17)$ & 0.01 \\
$2 \mathrm{~h}$ & $8.7(2.0)$ & $8.0(1.8)$ & & \\
\hline
\end{tabular}

BLISS: Breastfeeding Length Intensity Score System. BLISS [?]19: high intensity breastfeeding CI: confidence interval. Data given as mean (SD)

*Adjusted: Maternal age 2nd pregnancy, ethnicity, BMI, treatment of GDM in first pregnancy 